
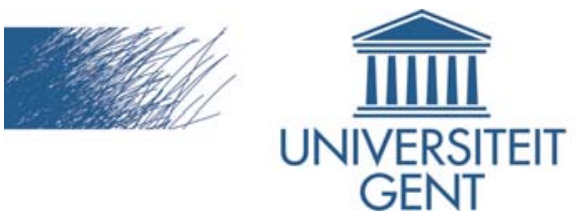

biblio.ugent.be

The UGent Institutional Repository is the electronic archiving and dissemination platform for all UGent research publications. Ghent University has implemented a mandate stipulating that all academic publications of UGent researchers should be deposited and archived in this repository. Except for items where current copyright restrictions apply, these papers are available in Open Access.

This item is the archived peer-reviewed author-version of: Mechanistic modelling of infrared mediated energy transfer during the primary drying step of a continuous freeze-drying process

Authors: Van Bockstal P.J., Mortier S., De Meyer L., Corver J., Vervaet C., Nopens I., De Beer T.

In: European Journal of Pharmaceutics and Bipharmaceutics 2017, 114: 11-21

To refer to or to cite this work, please use the citation to the published version:

Van Bockstal P.J., Mortier S., De Meyer L., Corver J., Vervaet C., Nopens I., De Beer T. (2017) Mechanistic modelling of infrared mediated energy transfer during the primary drying step of a continuous freeze-drying process

European Journal of Pharmaceutics and Bipharmaceutics 114: 11-21

DOI: 10.1016/j.ejpb.2017.01.001 


\title{
Mechanistic modelling of infrared mediated energy transfer during the primary drying step of a continuous freeze-drying process
}

\author{
Pieter-Jan Van Bockstal ${ }^{\mathrm{a}}$, Séverine Thérèse F.C. Mortier ${ }^{\mathrm{a}, \mathrm{b}}$, Laurens De Meyer ${ }^{\mathrm{a}}$, Jos Corver ${ }^{\mathrm{a}}$, Chris \\ Vervaet $^{\mathrm{c}}$, Ingmar Nopens ${ }^{\mathrm{b}}$, Thomas De Beer $\mathrm{r}^{\mathrm{a}, 1,2, *}$ \\ ${ }^{a}$ Laboratory of Pharmaceutical Process Analytical Technology, Department of Pharmaceutical Analysis, Faculty of \\ Pharmaceutical Sciences, Ghent University, Ottergemsesteenweg 460, 9000 Ghent, Belgium \\ ${ }^{b}$ BIOMATH, Department of Mathematical Modelling, Statistics and Bioinformatics, Faculty of Bioscience Engingeering, \\ Ghent University, Coupure Links 653, 9000 Ghent, Belgium \\ ${ }^{c}$ Laboratory of Pharmaceutical Technology, Department of Pharmaceutics, Faculty of Pharmaceutical Sciences, Ghent \\ University, Ottergemsesteenweg 460, 9000 Ghent, Belgium
}

\begin{abstract}
Conventional pharmaceutical freeze-drying is an inefficient and expensive batch-wise process, associated with several disadvantages leading to an uncontrolled end product variability. The proposed continuous alternative, based on spinning the vials during freezing and on optimal energy supply during drying, strongly increases process efficiency and improves product quality (uniformity). The heat transfer during continuous drying of the spin frozen vials is provided via non-contact infrared (IR) radiation. The energy transfer to the spin frozen vials should be optimized to maximise the drying efficiency while avoiding cake collapse. Therefore, a mechanistic model was developed which allows computing the optimal, dynamic IR heater temperature in function of the primary drying progress and which, hence, also allows predicting the primary drying endpoint based on the applied dynamic IR heater temperature. The model was validated by drying spin frozen vials containing the model formulation $(3.9 \mathrm{~mL}$ in $10 \mathrm{R}$ vials) according to the computed IR heater temperature profile. In total, 6 validation experiments were conducted. The primary drying endpoint was experimentally determined via in-line near-infrared (NIR) spectroscopy and compared with the endpoint predicted by the model (50 minutes). The mean ratio of the experimental drying time to the predicted value was 0.91 , indicating a good agreement between the model predictions and the experimental data. The end product had an elegant product appearance (visual inspection) and an acceptable residual moisture content (Karl Fischer).
\end{abstract}

Keywords: Continuous freeze-drying, Spin freezing, Infrared radiation, Mechanistic modelling

\section{Introduction}

Conventional pharmaceutical manufacturing is generally accomplished using inefficient batch processes

11. Batch-wise manufacturing is characterised by the processing of starting materials through a sequence of segmented unit operations, each with specific process settings, until the final product is obtained [2].

\footnotetext{
* Corresponding author

Email addresses: PieterJan.VanBockstal@UGent.be (Pieter-Jan Van Bockstal), Severine.Mortier@UGent.be (Séverine Thérèse F.C. Mortier), Laurens.DeMeyer@UGent.be (Laurens De Meyer), Jos.Corver@RheaVita.nl (Jos Corver), Chris.Vervaet@UGent. be (Chris Vervaet), Ingmar. Nopens@UGent.be (Ingmar Nopens), Thomas.DeBeer@UGent.be (Thomas De Beer )

${ }^{1}$ Phone number: $+32(0) 92648097$

${ }^{2}$ Fax number: +32(0)9 2228263
} 
The (off-line) quality assessment of intermediate products after each unit operation strongly delays the production process. Additionally, rejection or reprocessing of the entire batch is required in case product specifications are not met, which has a significant economical impact. With the aim of increasing the process efficiency, the pharmaceutical industry, also encouraged by the regulatory authorities, is currently shifting from batch-wise production processes to continuous manufacturing. In continuous processes, all unit operations are integrated in a single production line with continuous feeding of raw materials and the concomitant removal of finished products. In the development of continuous alternatives for batch-wise pharmaceutical manufacturing processes, the focus till now was mainly on solid dosage forms. However, continuous processing of biopharmaceuticals, both up- and downstream, is also of major importance [3]. Biopharmaceutical drug products often have a limited stability in aqueous solution due to several degradation pathways, mediated by water 4 . The preferred way of stabilising these products is by removing the aqueous solvent through freeze-drying (lyophilisation) [5. Conventional pharmaceutical freeze-drying is an inefficient batch-wise process, associated with several disadvantages leading to an uncontrolled end product variability [6] 8]. In this respect, an innovative freeze-drying concept resolving all disadvantages of batch freeze-drying, is proposed for the continuous processing of unit-doses $[9$.

The continuous freeze-drying process is initiated by rapidly rotating glass vials containing the aqueous drug formulation along their longitudinal axis [7, 8. The flow of a cold, inert and sterile gas cools the solution, followed by the induction of ice nucleation [9]. The formed ice crystals start growing upon further cooling, leading to a gradual increase in solute concentration [10. When a saturated solution is reached at the eutectic temperature $T_{e}$, some compounds such as mannitol, sodium chloride or glycine start to crystallize. For non-crystallising materials, the freeze-concentrate becomes supersaturated, associated with an increase in viscosity. This supersaturation of the freeze-concentrate continues until the viscosity has reached a level beyond which further ice crystallisation is inhibited. The temperature at which this maximum freezeconcentration occurs, is the glass transition temperature $T_{g}^{\prime}$. Because of the inhibition of ice crystallisation at $T_{g}^{\prime}$, a residual amount of unfrozen water is still present in the amorphous solid. At the end of the primary spin freezing step, the product is solidified over the entire inner vial wall resulting in a thin product layer. Subsequently, the spin frozen vials are continuously transferred to a long belt in a temperature-controlled chamber, which allows further crystallisation and solidification of the solutes under standardized conditions (annealing). After obtaining the desired morphological structure during this secondary freezing step, the spin frozen vials are further processed to the primary drying unit. Here, the pressure is kept at a constant value between 10 to $30 \mathrm{~Pa}$. Hence, an appropriate load-lock system is required for vial transfer between the 
freezing unit (at atmospheric pressure) and the drying unit. Continuous primary drying requires a uniform and adequate energy supply towards the entire vial surface to achieve an efficient and homogeneous drying behaviour. This energy transfer can be provided via conduction, by placing spin frozen vials in individual, close-fitting temperature-controlled pockets [7. Alternatively, a previous study showed the feasibility of IR radiation to provide the heat needed for drying of the spin frozen vials $[8]$. The $\mathrm{IR}$ mediated primary drying process is illustrated in figure 1 Each spin frozen vial is rotating along its longitudinal axis in front of an individual IR heater, which can be controlled at a specific temperature. Rotation of the vials is necessary to obtain a uniform heat transfer. Movement of the vials takes place in discrete steps ensuring that each vial is almost constantly centred in front of an individual IR heater. The application of non-contact IR radiation offers some advantages, relative to conduction. Monitoring the drying behaviour is facilitated in non-enveloped vials. Additionally, a whole range of vial types can be processed without the need to customise the heatable pockets to different vial dimensions.

During the last step of the continuous freeze-drying process, secondary drying, any remaining unfrozen water is removed by desorption until the final dried product with the desired residual moisture content is obtained. Depending on whether secondary drying requires a pressure level different from primary drying, a second continuous drying unit is necessary, separated from the first one by an appropriate load-lock system for vial transport 9. At the end of the continuous lyophilisation process, vials are removed from the vacuum conditions in the drying module via another load-lock system and transferred to the final unit, which allows the stoppering of the processed vials, in a controlled nitrogen environment.

Spin freezing of glass vials and the corresponding decrease in layer thickness strongly increases the drying efficiency compared to conventional batch freeze-drying. To obtain an as high as possible throughput, it is furthermore necessary to maximise the drying efficiency by providing sufficient radiation energy to the spin frozen vials during its entire drying process. On the other hand, product appearance is an important Critical Quality Attribute (CQA) of freeze-dried products and cake collapse should be avoided at all cost to ensure fast reconstitution of the dried product and for aesthetic purposes [10, 11]. Therefore, the product temperature during primary drying should never exceed $T_{e}$ or the collapse temperature $T_{c}$ of the formulation for crystalline or amorphous materials, respectively. In general, $T_{c}$ is a few degrees higher than $T_{g}^{\prime}$ because the viscosity near $T_{g}^{\prime}$ is still sufficiently high to prevent molecular motion [10. The thickness of the dried product layer increases with the primary drying progress. The dried product mass transfer resistance $R_{p}$ also raises, associated with a gradual increase in product temperature. To maintain the product temperature below the critical value during the entire process, the optimal radiation energy transfer to the spin frozen 
vial is rather dynamic instead of static.

Mechanistic modelling of the primary drying step in conventional batch freeze-drying has already been extensively described [12 17]. These models allow the computation of the adaptable process parameters, shelf temperature and chamber pressure, maximising the sublimation rate while preventing cake collapse. Additionally, inclusion of the uncertainty on each process and formulation parameter of the primary drying model leads to the construction of the (dynamic) Design Space for a specific lyophilized formulation, allowing qualitative assessment of the risk of cake collapse [18. Besides increasing process efficiency, mechanistic modelling also offers fundamental process knowledge which contributes to the understanding of the lyophilisation process.

In a former study, a Design of Experiments (DoE) approach was used to determine the impact of several radiation drying related process parameters on the drying behaviour of spin frozen formulations [8. However, no fundamental insight in the underlying physics of the process was obtained via this approach. In addition, the resulting empirical models are only valid for the different formulations and parameter ranges included in this DoE Because of both these reasons, it is clear that the continuous freeze-drying concept would benefit from mechanistic models (similar to conventional batch freeze-drying) which describe the primary drying behaviour of spin frozen vials. The aim of this paper is the development and validation of a mechanistic model which computes the optimal, dynamic IR heater temperature in function of the drying progress of a formulation in a spin frozen vial, maximising the drying efficiency while maintaining an elegant product structure. Additionally, the primary drying model allows the determination of the amount of sublimed ice at each time point and the computation of the primary drying endpoint.

\section{Materials and methods}

\subsection{Development of primary drying model for continuous drying of spin frozen vials via radiation}

Initially, the maximum allowable sublimation rate $\dot{m}_{\text {sub }}$ is calculated as several limitations need to be considered. First, $\dot{m}_{\text {sub }}$ is restricted by the dried product mass transfer resistance $R_{p}$ as only a limited amount of water vapour can escape the sublimation interface through the pores of the dried product. If more water vapour is generated at the sublimation interface, the mass transfer through the dried layer will be obstructed, leading to a local pressure increase at this interface. Since the product temperature at the sublimation front $T_{i}$ is in equilibrium with the vapour pressure $P_{w, i}$, naturally, $T_{i}$ will also increase. However, during the entire primary drying process, $T_{i}$ should be maintained below the critical value, $T_{e}$ or $T_{c}$, to avoid collapse of the product. Therefore, $\dot{m}_{\text {sub }}$ is limited to a maximum to avoid that the critical 
product temperature $T_{i, c r i t}$ is exceeded during the primary drying stage. Consequently, as ice sublimation is an endothermic process, the energy transfer during primary drying is also limited.

The sublimation rate during primary drying $\dot{m}_{\text {sub }}(\mathrm{kg} / \mathrm{s})$ is calculated by:

$$
\dot{m}_{\mathrm{sub}}=\frac{A_{p}\left(P_{w, i}-P_{w, c}\right)}{R_{p}}
$$

where $A_{p}$ is the product area available for sublimation $\left(\mathrm{m}^{2}\right), P_{w, i}$ the vapour pressure of ice at the sublimation interface $(\mathrm{Pa}), P_{w, c}$ the partial pressure of water in the drying unit $(\mathrm{Pa})$ and $R_{p}$ the dried product mass transfer resistance $(\mathrm{m} / \mathrm{s}) . P_{w, c}$ is assumed to be equal to the overall pressure in the drying unit $P_{c}$, as during primary drying the gas composition in the drying unit consists almost entirely of water vapour, similar to batch freeze-drying [16].

$P_{w, i}$ is calculated by the following empirical equation [19]:

$$
P_{w, i}=133.3222 .710^{10} e^{-\frac{6145}{T_{i}}}
$$

where $T_{i}$ is the product temperature at the sublimation interface $(\mathrm{K})$. To maximise the drying efficiency and simultaneously avoid loss of cake structure, $T_{i}$ is set at the critical product temperature $T_{i, c r i t}$ $R_{p}$ depends on the thickness of the dried product layer $l(\mathrm{~m})$. This relation is given by the following empirical equation:

$$
R_{p}=R_{p, 0}+\frac{A_{R_{p}} l}{1+B_{R_{p}} l}
$$

where $R_{p, 0}(\mathrm{~m} / \mathrm{s}), A_{R_{p}}(1 / \mathrm{s})$ and $B_{R_{p}}(1 / \mathrm{m})$ are constants, determined by the fitting of experimental data [17. $R_{p}$ is formulation specific and is strongly influenced by the pore size of the dried product, which is mainly determined by the degree of supercooling during the freezing step [20, 21]. In general, $R_{p}$ increases with the primary drying progress and the corresponding increase in $l$. As a consequence, the energy input needs to be decreased so all generated water vapour can escape the sublimation front to avoid an increase in $P_{w, i}$ and $T_{i}$, associated with a decrease in $\dot{m}_{\text {sub }}$ (Equation (1)).

Spin freezing leads to a thin product layer, uniformly spread over the entire inner vial wall (Figure 1). Hence, $A_{p}$ is calculated by:

$$
A_{p}=2 \pi\left(r_{p, i}+l\right) h
$$


with $h$ the height of the spin frozen product $(\mathrm{m})$ and $r_{p, i}$ the radius from the centre of the vial to the border of the spin frozen layer $(\mathrm{m})$, which is given by:

$$
r_{p, i}=\sqrt{r_{v, i}^{2}-\frac{V}{\pi h}}
$$

where $r_{v, i}$ is the inner radius of the glass vial $(\mathrm{m})$ and $V$ is the filling volume $\left(\mathrm{m}^{3}\right)$. During primary drying, the sublimation front gradually moves from the inside of the vial towards the vial wall. For this reason, $A_{p}$ increases simultaneously with the increase of $l$.

The increase in the dried layer thickness $\Delta l(\mathrm{~m})$ for a specified time interval $\Delta t(\mathrm{~s})$ is calculated by:

$$
\Delta l=\frac{\dot{m}_{\mathrm{sub}} \Delta t}{A_{p} \rho_{i c e} \phi}
$$

with $\rho_{\text {ice }}$ the density of ice $\left(\mathrm{kg} / \mathrm{m}^{3}\right)$ and $\phi$ the volume fraction of ice (-).

Secondly, the maximum value of $\dot{m}_{\text {sub }}$ is limited by the choked flow phenomenon [22]. The gas flow rate to the condenser is restricted by an upper limit near the narrowest point in the processing equipment. In batch freeze-drying, the duct connecting the drying chamber with the condenser is considered the most critical point where choked flow can occur. In continuous freeze-drying, the amount of vials being processed at the same time is much smaller, which makes the occurrence of choked flow at the duct in the continuous drying unit unlikely. On the other hand, spin freezing results in a significant increase of $A_{p}$ in comparison with batch freeze-drying, which allows a much higher sublimation rate in each individual vial. For this reason, it is necessary to evaluate the risk of choked flow at the vial neck, being the narrowest point during sublimation. Choked flow occurs once the vapour flow rate through the vial neck approaches the speed of sound (Mach I), where a further increase in mass flow would result in an elevated vapour density, leading to a local pressure increase inside the vial. The difference between $P_{w, i}$ and $P_{w, c}$ becomes smaller, resulting in a decrease of the sublimation rate (Equation (1)), while the energy input is maintained at the same level. Consequently, more energy is provided to the spin frozen vial than can be removed through ice sublimation (maximum $R_{p}$ ), resulting in an increase in $T_{i}$. Eventually, $T_{i}$ will exceed $T_{i, c r i t}$, leading to loss of cake structure and rejection of the product. In general, choked flow is most likely to occur during the processing of a formulation characterised by a high value for $T_{i, c r i t}$ or a low $R_{p}$ profile, especially at the onset of primary drying.

Evaluation of the choked flow criterion requires the calculation of the speed of sound $c(\mathrm{~m} / \mathrm{s})$ for an ideal 
gas 22:

$$
c=\sqrt{\frac{\gamma R T_{r}}{M}}
$$

with $\gamma$ the ratio of specific heats (adiabatic index) for water (1.33), $R$ the gas constant $(8.31 \mathrm{~J} /(\mathrm{K} \mathrm{mol})$ ), $T_{r}$ the temperature of the water vapour $(\mathrm{K})$ and $M$ the molecular weight of water $(0.018 \mathrm{~kg} / \mathrm{mol})$. The maximum sublimation rate avoiding choked flow $\dot{m}_{\mathrm{sub}, \text { chok }}(\mathrm{kg} / \mathrm{s})$, is given by [23]

$$
\dot{m}_{\mathrm{sub}, \mathrm{chok}}=c \pi r_{v, n}^{2} \rho_{w v}
$$

with $r_{v, n}$ the radius of the vial neck $(\mathrm{m})$ and $\rho_{w v}$ the density of water vapour $\left(\mathrm{kg} / \mathrm{m}^{3}\right)$, which is given by the following equation for the calculation of the density of an ideal gas 23]:

$$
\rho_{w v}=\frac{P_{c} M}{T_{r} R}
$$

After computing the maximum allowable sublimation rate, the radiation energy required to support this sublimation rate $\dot{m}_{\text {sub }}$ is calculated by:

$$
P_{t o t}=\frac{\dot{m}_{\mathrm{sub}} \Delta H_{s}}{M}
$$

where $P_{t o t}$ is the total power that should be provided to the spin frozen vial (W) and $\Delta H_{s}$ is the latent heat of ice sublimation ( $51139 \mathrm{~J} / \mathrm{mol})$. The system was assumed to be at steady-state, i.e., the radiation energy was only used for ice sublimation.

It should be taken into account that a significant part of the required power is provided by the surroundings, as the walls and door of the vacuum chamber are at a higher temperature than the spin frozen vial. Therefore, $P_{t o t}$ is compensated for the radiation energy contributed by these surrounding surfaces:

$$
P_{\text {rad }}=P_{\text {tot }}-P_{\text {sur }}
$$

where $P_{\text {rad }}$ is the power that should be provided by the IR heater to the spin frozen vial $(\mathrm{W})$ and $P_{\text {sur }}$ is the power provided by the surroundings to the spin frozen vial (W).

Both $P_{\text {sur }}$ and the temperature of the IR heater necessary to provide sufficient energy to achieve the optimal sublimation rate are calculated via the Stefan-Boltzmann law:

$$
P=A_{\text {rad }} F \sigma\left(\epsilon T_{r a d}^{4}-a T_{v}^{4}\right)
$$


where $A_{\text {rad }}$ is the area of the emitting surface (i.e., IR heater or chamber wall) $\left(\mathrm{m}^{2}\right), F$ is the view factor $(-), \sigma$ the Stefan-Boltzmann constant $\left(5.6710^{-8} \mathrm{~W} /\left(\mathrm{m}^{2} \mathrm{~K}^{4}\right)\right), \epsilon$ is the emission coefficient of the emitting surface (-), $T_{\text {rad }}$ is the temperature of the emitting surface $(\mathrm{K}), a$ is the absorptivity of the target surface $(-)$ and $T_{v}$ is the temperature of the spin frozen vial $(\mathrm{K}) . P$ is equal to $P_{r a d}$ when the IR heater is the emitting surface or $P_{\text {sur }}$ when the chamber wall or door is the emitting surface. In general, $a$ is estimated as the value of $\epsilon$ for the target surface [23]. $T_{v}$ is assumed to be equal to $T_{i} . F$ is defined as the percentage of total radiation which leaves a specific surface and goes directly to the target surface [24. Both the IR heater and the walls of the drying chamber are considered diffuse emitters, meaning, these surfaces emit radiation uniformly in all directions. Consequently, $F$ only depends on the relative geometric orientation of the emitting surface (i.e., IR heater or chamber wall) to the spin frozen vial, represented by a flat plate and a cylinder, respectively. The computation of $F$ for this specific situation is based on the Monte Carlo method described by Brenner et al. 25]. The Monte Carlo method is a simulation approach in which a defined number of rays is propagated from random positions on the emitting surface at randomly chosen angles [24]. For each generated ray, it is evaluated whether it will directly hit the target surface or not. $F$ is estimated by the ratio of the number of rays that strike the target surface to the total amount of emitted rays.

\subsection{Temperature calibration of the $I R$ heaters}

The temperature of the IR heater $T_{\text {rad }}$ was determined in function of electric power $P_{e}$, delivered by the Voltcraft PPS-11360 power supply (Conrad Electronic, Hirschau, Germany). A $10 \mathrm{~mL}$ type I glass vial (Schott, Müllheim, Germany) filled with $3.9 \mathrm{~mL}$ of water was spin frozen according to the procedure described by Van Bockstal et al. 8]. The glass vial was placed in a vial holder and vertically rotated along its longitudinal axis at approximately 2900 rotations per minute (rpm). Consequently, the liquid was spread uniformly across the entire vial wall. The rotating vial was then immersed into liquid nitrogen for $40 \pm 5 \mathrm{~s}$ to completely solidify the product. Within $15 \pm 5 \mathrm{~s}$ after spin freezing, the vial was transferred from the liquid nitrogen to the drying chamber of an Amsco FINN-AQUA GT4 freeze-dryer (GEA, Köln, Germany), herewith avoiding exceedance of the melting point of ice. The shelves in the drying chamber were set at a constant temperature of $-10^{\circ} \mathrm{C}$. The spin frozen vial was presented to one IR heater (Weiss Technik, Zellik, Belgium) at a distance of $4 \mathrm{~cm}$ measured from the centre of the vial until the heated filament of the IR heater. No contact was made with the shelf. The vial was continuously rotating at $5 \mathrm{rpm}$ which was necessary for a homogeneous energy transfer from the IR heater to the vial. Immediately after placing the vial in the drying chamber, the pressure was lowered to $13.3 \mathrm{~Pa}$. Within 5 minutes, the pressure in the 
drying chamber was below the triple point of water, hence avoiding any melting of ice. After exactly 17 minutes, the time necessary to achieve the desired pressure level, the IR heaters were activated. During each individual experiment, primary drying was conducted at a different level of $P_{e}(7 \mathrm{~W}, 18 \mathrm{~W}, 32 \mathrm{~W}, 49 \mathrm{~W}$ and $69 \mathrm{~W}$ ), provided by the power supply. The temperature of the wall of the glass vial was continuously monitored with a type-K thermocouple (Conrad Electronic, Hirschau, Germany). The drying chamber was aerated with nitrogen 20 min after activation of the IR heaters to interrupt ice sublimation after which the sublimed ice mass was immediately determined gravimetrically, including a correction for the amount of ice sublimed during the introduction of the vacuum in the drying chamber and for the computed value of $P_{\text {sur }}$, as described in section 2.3. $T_{\text {rad }}$ is calculated with the help of equations (10), (11) and (12) in function of $P_{e}$. In addition, using the same equations, the efficiency of the $\mathrm{IR}$ heater $\eta$ was calculated by taking the ratio of the net power provided to the spin frozen vial $P_{r a d}$ and $P_{e}$ :

$$
\eta=\frac{P_{r a d}}{P_{e}}
$$

Linear regression using the least squares approach was applied to describe $T_{\text {rad }}$ in function of $P_{e}$. Both a linear and a quadratic empirical model were developed to describe this relation. The significance of the regression coefficients of both equations was statistically evaluated via a $t$-test $(\alpha=5 \%)$ using SPSS (Version 23.0, IBM, Armonk, IL, USA). For each regression coefficient the null hypothesis, stating that a coefficient was equal to zero, was tested. Acceptance of the null hypothesis for a specific regression coefficient indicated that the corresponding term was not significant. In addition, the Root Mean Square Error of Cross-Validation (RMSECV) was calculated for the linear and quadratic model to compare the predictive power of both models.

\subsection{Verification of radiation energy contributed by the surroundings}

The power radiated by the surroundings was calculated individually for each plane surface contributing to the IR energy transfer to the spin frozen vial: the acrylic glass door, the three stainless steel chamber walls, the stainless steel shelf located above and below the experimental set-up and the inactivated IR heater. The view factor $F$ was computed for each of these surfaces via Monte Carlo simulation to determine the fraction of radiation which reaches the spin frozen vial [25]. A defined number of rays is propagated from randomly chosen positions on the emitting surface (i.e., the door, the chamber walls, the shelf and the IR heater) at random angles. For each simulated ray, the conditions are evaluated whether the target surface (i.e., the spin frozen vial) is hit or not. $F$ is equal to the ratio of the number of hits to the amount of simulated rays. The 
net power contribution was then calculated with the help of equations 100 and 12 . The nominal values of the model parameters required for the computation of both the view factor and the power contributed by the surroundings are listed in table 1 . The computed IR energy contribution radiated by the surroundings to the spin frozen vial was verified with experimental data. A $10 \mathrm{~mL}$ type I glass vial filled with $3.9 \mathrm{~mL}$ of water was spin frozen and transferred to the drying chamber as described in section 2.2 The amount of ice sublimed during the initial pressure decrease, i.e. the 17 minutes lasting period immediately after activation of the vacuum pump, was determined gravimetrically. In a second experiment, the vacuum was maintained for another 20 minutes after reaching the desired pressure $(13.3 \mathrm{~Pa})$, i.e. 37 minutes in total, without activation of the $\mathrm{IR}$ heater. After this period, the sublimed ice mass was determined gravimetrically. Both experiments were conducted in triplicate. With the help of equation $\sqrt{10}$, the power corresponding to this sublimation rate was calculated. The difference in power between both experiments reflects the energy provided through radiation from the surroundings to the spin frozen vial.

\subsection{Model validation}

The primary drying model computing the IR mediated heat transfer to the spin frozen vials was validated for a model formulation. This aqueous formulation contained $30 \mathrm{mg} / \mathrm{mL}$ mannitol, $3.42 \mathrm{mg} / \mathrm{mL}$ sucrose, $3.75 \mathrm{mg} / \mathrm{mL}$ glycine and $0.58 \mathrm{mg} / \mathrm{mL}$ sodium chloride. Mannitol, sucrose and sodium chloride were purchased from Fagron (Waregem, Belgium). Glycine was acquired from Sigma-Aldrich (Saint Louis, MO, USA). The optimal dynamic IR heater temperature profile in function of primary drying time $t$ was computed as described in section 2.1. Similar as for the IR heater calibration, a correction was made for the amount of ice sublimed during the initial pressure decrease (Section 2.2. The nominal values of the model parameters required for solving the model equations are listed in Table 1. The parameters describing $R_{p}$ in function of

$l$ were taken from literature [21]. $T_{i, \text { crit }}$ was assumed to be equal to $T_{g}^{\prime}$ of the model formulation. As the model formulation was low concentrated, $\phi$ was assumed to be equal to 1 . The time step $\Delta t$ was set at $60 \mathrm{~s}$. The calibration of the IR heaters allowed calculating the dynamic $P_{e}$ profile corresponding to the optimal dynamic IR heater temperature profile.

A $10 \mathrm{~mL}$ type I glass vial (Schott, Müllheim, Germany) filled with $3.9 \mathrm{~mL}$ of the model formulation was spin frozen according to the procedure described in section 2.2 . The volume of $3.9 \mathrm{~mL}$ corresponded to a thickness of the frozen product layer of $1.63 \mathrm{~mm}$. The applied vertical rotation speed of $2900 \mathrm{rpm}$ resulted in a homogeneous product layer with a maximum deviation of the layer thickness from top to bottom of 
$4.81 \%$ as calculated by:

$$
\Delta L_{t o t}=\frac{h g}{2 \pi \omega^{2} r_{p, i}}
$$

with $\Delta L_{t o t}$ the deviation to the average thickness of the spin frozen layer $(\mathrm{m}), g$ the gravitational acceleration $\left(9.81 \mathrm{~m} / \mathrm{s}^{2}\right)$ and $\omega$ the angular velocity $(\mathrm{rad} / \mathrm{s})$. After spin freezing, the vial was transferred to the drying chamber where primary drying was conducted as described in section 2.2 . The experimental drying set-up is shown in figure 2. When the desired pressure (13.3 Pa) was achieved, 17 minutes after activation of the vacuum pump, the preprogrammed $P_{e}$ profile was initiated. At the end of the dynamic profile, the drying process was prolonged by maintaining the IR heater temperature corresponding to the last value of $P_{e}$ of this profile for 30 minutes to ensure that all ice was sublimed. This validation experiment was repeated 6 times. The entire drying process was continuously monitored with in-line NIR spectroscopy with the aim of determining the primary drying endpoint $[8]$.

\subsection{Determination of the critical product temperature}

The critical product temperature $T_{i, \text { crit }}$ of the model formulation during primary drying was determined via Modulated Differential Scanning Calorimetry (MDSC) using a differential scanning calorimeter Q2000 (TA instruments, Zellik, Belgium). Hermetically sealed aluminium pans (TA instruments, Zellik, Belgium) were filled with approximately $12 \mathrm{mg}$ of the formulation. The DSC cell was constantly purged with dry nitrogen at a flow rate of $50 \mathrm{~mL} / \mathrm{min}$. The sample was initially cooled until $-90{ }^{\circ} \mathrm{C}$. This temperature was maintained for 5 minutes. Subsequently the temperature was linearly increased until $0{ }^{\circ} \mathrm{C}$ at a heating rate of $2{ }^{\circ} \mathrm{C} / \mathrm{min}$. The modulation amplitude and period were set at $0.212{ }^{\circ} \mathrm{C}$ and 40 seconds, respectively. The analysis was conducted in triplicate. The thermograms were analysed with TA Instruments Universal Analysis 2000 version 4.7A (TA Instruments, Zellik, Belgium).

\subsection{NIR spectroscopy}

Diffuse reflectance NIR spectra were continuously in-line collected with an Antaris ${ }^{\mathrm{TM}}$ II Fourier-Transform NIR spectrometer (Thermo Fisher Scientific, Erembodegem, Belgium), equipped with a quartz halogen lamp, a Michelson interferometer and an InGaAs detector [8]. The fibre optic probe was implemented in the drying chamber at a distance of $0.5 \pm 0.1 \mathrm{~mm}$ near the middle of the vial without hampering or disturbing the rotation of the vial (Figure 22. As drying progresses from the centre of the vial to the inner vial wall, in-line NIR spectroscopy allowed the detection of complete ice removal, i.e. the endpoint of primary drying. Every 20 seconds a NIR spectrum was collected in the $4500-10000 \mathrm{~cm}^{-1}$ region with a resolution of $16 \mathrm{~cm}^{-1}$ and 
averaged over 4 scans. The illumination spot size obtained with the NIR probe was approximately $28 \mathrm{~mm}^{2}$. Due to rotation of the vial during the measurements, each spectrum was collected at a different position of the cake on a specific height. It was assumed that this monitored part is representative for the whole cake.

\subsection{Multivariate data analysis}

The collected NIR spectra during each validation run were analysed with the help of the multivariate data analysis software SIMCA (Version 14.0.0, Umetrics, Umeå, Sweden) [8]. The NIR spectra collected before activation of the heaters were removed from each dataset. The Savitzky-Golay filter was applied to smooth the spectra: a quadratic polynomial function was fitted to a moving sub-model, each containing fifteen data points. Additionally, Standard Normal Variate (SNV) preprocessing was applied to eliminate the additive baseline offset variations and multiplicative scaling effects in the spectra which may be caused by small variations in distance between the NIR probe and the rotating glass vial and possible differences in product density [26]. Principal Component Analysis (PCA) was then used for the analysis of the preprocessed and mean-centred NIR spectra.

PCA is an unsupervised multivariate projection method which extracts and displays the variation in the data set [27, 28]. The original variables, e.g. the individual wave numbers of the NIR spectra, are replaced by a new set of latent variables, named principal components (PCs), These PCs are sequentially acquired by an orthogonal, bilinear decomposition of the data matrix. Each component explains most of the remaining variability in the data. $\mathrm{PCs}$ are composed of a score and a loading vector. The score vector contains a score value for each spectrum, which describes its quantitative relation to the other spectra. The loading vector provides qualitative information about which spectral features present in the original observations are captured by the corresponding component.

\subsection{Karl Fischer}

The residual moisture content of the freeze-dried product obtained from the model validation runs, was determined using Karl Fischer titration as described by Hansen et al. 29. The freeze-dried product was reconstituted with a known volume of dry methanol (Sigma-Aldrich, Saint Louis, MO, USA) and left to equilibrate for roughly 15 minutes. With the help of a syringe, a known volume of this solution was volumetrically removed from the vial, injected in the titration vessel of the Mettler Toledo V30 volumetric Karl Fischer titrator (Schwerzenbach, Switzerland) and titrated with Hydranal ${ }^{\circledR}$ titration solvent (SigmaAldrich, Saint Louis, MO, USA). Prior to the measurement of the residual water, the moisture content of the 
dry methanol was determined in triplicate and subtracted from the result. The residual moisture content of the freeze-dried product was determined for three different validation runs.

\section{Results and Discussion}

\subsection{IR heater calibration}

The mass of sublimed ice $m(\mathrm{~g})$ after 20 minutes of drying and corrected for the energy contributed by the surroundings and the corresponding $\mathrm{IR}$ heater temperature $T_{\text {rad }}$ (as calculated from equations 10 ) and $(12)$ ) are both plotted in function of the applied electric power $P_{e}$ in figure 3 Initially, a linear model was fitted, though, the parabolic distribution of the standardized residuals of the linear model suggested that a quadratic model might be preferred to explain this relation (standardized residual plot not shown). Therefore, a $t$-test $(\alpha=5 \%)$ was conducted to evaluate the statistical significance of the regression coefficients of both the linear and quadratic model. The $t$-value $(-2.474)$ for the regression coefficient associated with the quadratic term lies between the critical values -3.182 an 3.182 of the $t$ distribution (degrees of freedom $=$ 3 ) and the corresponding $p$-value (0.132) is higher than the significance level $\alpha$. Hence, the null hypothesis is not rejected which means that this regression coefficient is not significant at the $5 \%$ significance level. Therefore, a linear model is sufficient (from which all regression coefficients are proven to be significant). In addition, RMSECV for the linear and quadratic model was $35.90 \mathrm{~K}$ and $38.67 \mathrm{~K}$, respectively. Hence, the predictive power of the linear model is higher compared to the quadratic model. Based on these findings, the relation between $T_{r a d}$ and $P_{e}$ was described by the following (empirical) linear equation, plotted in figure 3

$$
T_{\text {rad }}=5.120 P_{e}+425.173
$$

Comparison of $P_{e}$ and the net power provided to the spin frozen vial $P_{\text {rad }}$ allowed the determination of the IR heater efficiency $\eta$ (Equation (13)). The mean value of $\eta$ was $6.61 \%$. However, this is not the efficiency inherent to the IR heater itself as only part of the emitted radiation reaches the spin frozen vial. Therefore, it was necessary to include the view factor $F$ in the calculations. $F$ is defined as the percentage of total radiation which leaves the $\mathrm{IR}$ heater and goes directly to the spin frozen vial [24]. In the current experimental set-up, the vial was located in the middle of the IR heater at a distance of $4 \mathrm{~cm}$, measured from the centre of the vial. The corresponding value of $F$ was 0.1484 . Hence, the value of $\eta$ inherent to the $\mathrm{IR}$ heater is $44.54 \%$. The distance between the IR heater and the spin frozen vial $(s)$ has a major impact on $F$, as illustrated in figure 4. $F$ is subject to an exponential decay with the increase of $s . \eta$ exhibits the 
same behaviour. Consequently, in order to maintain an equal net energy transfer to the spin frozen vial, $P_{e}$ needs to be adapted proportionally to the change in $s$.

\subsection{Verification of radiation contribution from surroundings}

The ice mass sublimed during the verification runs with inactivated heaters was found to be $0.543 \pm 0.0395 \mathrm{~g}$ $\left(\dot{m}_{\text {sub }}=4.5310^{-7} \mathrm{~kg} / \mathrm{s}\right)$. This value had to be compensated for the amount of ice sublimed during the initial pressure decrease in the drying chamber, which was $0.219 \pm 0.00251 \mathrm{~g}$. Hence, the net sublimed ice mass was $0.324 \pm 0.0539 \mathrm{~g}\left(\dot{m}_{\text {sub }}=2.7010^{-7} \mathrm{~kg} / \mathrm{s}\right)$ which corresponds to an experimental $P_{\text {sur }}$ of $0.766 \pm 0.128 \mathrm{~W}$. The computed value of $P_{\text {sur }}$ was $0.649 \mathrm{~W}$, associated with a theoretical $\dot{m}_{\text {sub }}$ of $2.2910^{-7} \mathrm{~kg} / \mathrm{s}$. The power contribution of the surroundings is hence slightly underestimated by the model, with an absolute and relative difference of $0.117 \mathrm{~W}$ and $18.0 \%$, respectively. A reason for this underestimation might be that the model only includes the six plane surfaces (the chamber walls and the door of the drying chamber and the shelves above and below the experimental set-up) and the inactive heater in the calculation of the radiation contributed by the surroundings. To reduce the complexity of the model, the parts of the experimental set-up (aluminium frame etc.) were not taken into account which might be responsible for the difference between the experimental and computed $P_{\text {sur }}$. Finally, the absolute value of $P_{\text {sur }}$ is very low in comparison to $P_{t o t}$. Therefore, it can be considered that the model provides a good approximation of the radiation energy contributed by the surroundings to the spin frozen vial.

\subsection{Mechanistic primary drying model predictions}

The mechanistic primary drying model (Section 2.1) computes the optimal dynamic IR heater temperature profile for the most efficient primary drying without exceeding $T_{i, c r i t} . T_{\text {rad }}$ and $l$ are both plotted in function of the primary drying progress $t$ (Figure 5). At the start of the drying process by the heaters, $l$ is predicted to be different from zero, since a significant amount of ice already instantly sublimed during the initial pressure decrease $(0.167 \pm 0.0143 \mathrm{~g})$. After 50 minutes of primary drying, $l$ is predicted to be equal to the total layer thickness $L_{t o t}$ indicating complete ice removal. At the beginning of the process $T_{\text {rad }}$ is $728 \mathrm{~K}$, while it slowly decreases until $610 \mathrm{~K}$ towards the end of drying. As $R_{p}$ gradually raises with the increase of $l$, the energy input should be reduced accordingly with the progress of primary drying, to avoid exceedance of $T_{i, \text { crit }}$ [16]. Therefore, the model predicts $T_{\text {rad }}$ to be gradually decreasing as primary drying proceeds. Via equation (15), the corresponding $P_{e}$ profile can be calculated, which is to be used as input for the IR heaters allowing to put the model into practice. 


\subsection{Experimental model validation}

Each experimental model validation run $(\mathrm{n}=6)$ was continuously monitored with in-line NIR spectroscopy. Via $\mathrm{PCA}$ of the preprocessed $\mathrm{NIR}$ spectra, the $\mathrm{PCl}$ vs $\mathrm{PC}$ scores scatter plot was constructed per validation experiment, as illustrated in figure 6 for one individual experiment. A significant part of the spectral variability $(91.40 \%)$ was explained by $\mathrm{PCl}$, a smaller part of the variability was explained by PC2 (6.95\%). In the loading line plots of both $\mathrm{PCS}$, some NIR bands were identified (Figure 7). The band near $5000 \mathrm{~cm}^{-1}$ directed downwards in $\mathrm{PC} 1$ and upwards in $\mathrm{PC} 2$ stems from $\mathrm{O}-\mathrm{H}$ stretching and $\mathrm{H}-\mathrm{O}-\mathrm{H}$ bending vibrations of ice 30 . The broad band near $6700 \mathrm{~cm}^{-1}$ directed downwards in $\mathrm{PC} 1$ and upwards in PC 2 originates from symmetric and asymmetric stretch of ice [30. Therefore, the loading line plots clearly represent the changes in ice content in the monitored region of the spin frozen vial. These plots assisted in the full explanation of the changing $\mathrm{NIR}$ spectra in the scores plot, allowing the determination of the primary drying endpoint 8$]$.

At the beginning of primary drying, the NIR spectra were completely dominated by overwhelming ice bands. Therefore, the scores of the corresponding NIR spectra were initially clustered in the scores plot (Figure 6). At this point, both ice and product were present in the spin frozen vial. With the progression of primary drying, the scores move gradually upwards in the positive direction along PC2 (Figure 6). The bands in the NIR spectra near $5030 \mathrm{~cm}^{-1}$ and $6700 \mathrm{~cm}^{-1}$ become more distinct in shape NIR spectra not shown), confirmed by the loading line plot of $\mathrm{PC} 2$ where both bands are directed upwards (Figure 7). Ice removal proceeds from the centre of the vial in the direction of the glass wall, while NIR spectra were collected from the outer side. The NIR light only has a limited penetration depth into the sample. Hence, ice signals kept dominating the NIR spectra. However, the progress in ice sublimation and the change in the NIR spectra are clearly visible in the scores plot. This trend is probably associated with the reduction in ice layer thickness [8]. Only after the inflection point in the scores plot (Figure 6), characteristic product bands started to appear in the NIR spectra, besides ice specific bands which were still present, but with a lower intensity (NIR spectra not shown). The ice signals only completely disappeared in the NIR spectra in the cluster of scores, situated on the right in the scores plot, illustrated by the characteristic ice bands directed downwards in the loadings of $\mathrm{PCl}$ (Figure 6 and 7 ). At this stage, primary drying was completely finished and the final dried product was achieved. In contrast to amorphous products, no removal of residual moisture occurred as the model formulation resulted in a mostly crystalline product identified as $\beta$ mannitol via Raman spectroscopy, without any traces of mannitol hemihydrate (Raman spectra not shown).

The spin frozen vial was constantly rotating during primary drying. Hence, each NIR spectrum was col- 
lected at a random position at the monitored height of the vial. Despite the homogeneous energy transfer towards the spin frozen vial, primary drying was locally finished at slightly different time points across the monitored section, which was reflected in the score plot. Primary drying was only considered completely finished, when all ice was sublimated at each position of the monitored height of the spin frozen vial. The experimental primary drying time was defined as the time between the recording of the first spectrum after the onset of the $\mathrm{IR}$ heaters and the spectrum following the last spectrum situated outside the cluster in the score plot (Figure 6).

For each validation run, the scores scatter plot exhibited the same trends. Each scores plot was analysed as described above to determine the experimental drying time. Comparison was made between the computed and the experimental primary drying time. The results are listed in table 2 The experimental drying time was consistent for all repeated runs with an absolute difference of only 3.5 minutes between the two extremes. In addition, there was a fairly good agreement between the predicted (based on the developed model) and the experimentally determined primary drying time, as the mean ratio of both was $0.91 \pm 0.025$. The mechanistic model slightly overestimated the primary drying time as for each experiment sublimation was finished before the computed endpoint. This slight deviation could be explained by several hypotheses. Verification of the radiation energy contributed by the surroundings lead to the conclusion that the experimental energy transfer from the chamber walls to the spin frozen vial was higher than estimated by the model (Section 3.2). Although the absolute difference was very small, this is a possible reason for the faster primary drying time that was observed. The inertia of the IR heater is another hypothesis that could cause the small deviation between prediction and reality. $T_{\text {rad }}$ is gradually decreased with the progress of primary drying to limit the energy transfer due to the increase in $R_{p}$. The power input to the $\mathrm{IR}$ heater is lowered in discrete steps until a new level of the computed profile. However, the radiator can not immediately lose all the excess energy at once. $T_{\text {rad }}$ decreases steadily until the new equilibrium value is reached, which is associated with a small delay in the response of the IR heater to the power input during the process. This higher value for $T_{\text {rad }}$ during (a part of) primary drying might contribute to the higher energy transfer to the spin frozen vial. Finally, some input variables and process parameters of the primary drying model, such as $R_{p}$ or $T_{r a d}$, are an estimation of the actual value. Inherently, these factors are associated with a certain measurement error. For instance, this was illustrated for $T_{\text {rad }}$ via the calculation of RMSECV (Section 3.1). This might be the most plausible hypothesis to explain the small deviation between the computed model prediction of the primary drying time and the experimental observations.

Drying of the spin frozen vials following the computed $T_{\text {rad }}$ profile resulted in an elegant product appearance 
without any signs of collapse, visualised in figure 8 . Finally, the residual moisture content of the dried end product was determined via Karl Fischer titration. The remaining unfrozen water concentration was found to be $0.99 \pm 0.28 \%$, which is near the standard target of approximately $1 \%$ for batch freeze-dried products 31.

\section{Conclusion and future perspectives}

The developed mechanistic primary drying model allows the computation of the optimal, dynamic IR heater temperature profile, leading to the optimization of the drying efficiency of spin frozen vials. The validation experiments showed a good agreement between the experimental primary drying time and the model predictions $(0.91 \pm 0.25)$. In-line $\mathrm{NIR}$ spectroscopy allowed the determination of the primary drying endpoint during the model validation experiments. The dried end product had an elegant product appearance and an acceptable residual moisture content $(0.99 \pm 0.28 \%)$.

During the development of the mechanistic primary drying model, inherently, a few assumptions and simplifications were included. Additionally, some input variables and process parameters are an estimation of the actual value, associated with a certain measurement error. These uncertainties propagate to the model output, which might explain the small deviation between the experimental primary drying time and the model predictions. Similar to the primary drying models developed for batch freeze-drying, an uncertainty analysis will be conducted in a follow-up study to clarify this relatively small deviation. The uncertainty analysis will estimate and quantify the uncertainty for each input variable and process parameter in the model, leading to the quantification and control of the risk of cake collapse for the resulting dynamic IR heater profile.

\section{Nomenclature}

$\Delta H_{s} \quad$ Latent heat of ice sublimation $(51139 \mathrm{~J} / \mathrm{mol})$

$\Delta l \quad$ Increase in dried product layer per time step $\Delta t(\mathrm{~m})$

$\Delta L_{t o t}$ Deviation to the average thickness of the spin frozen layer (m)

$\Delta t \quad$ Time step to calculate $\Delta l(\mathrm{~s})$

$\dot{m}_{\text {sub,chok }}$ Maximum sublimation rate avoiding choked flow $(\mathrm{kg} / \mathrm{s})$

$\dot{m}_{\text {sub }}$ Sublimation rate $(\mathrm{kg} / \mathrm{s})$ 
$\epsilon \quad$ Emission coefficient of the emitting surface (-)

$\eta \quad$ Efficiency of IR heater (-)

$\gamma \quad$ Ratio of specific heats (-)

$\omega \quad$ Angular velocity ( $\mathrm{rad} / \mathrm{s})$

$\phi \quad$ Volume fraction of ice (-)

$\rho_{\text {ice }} \quad$ Density of ice $\left(\mathrm{kg} / \mathrm{m}^{3}\right)$

$\rho_{w v} \quad$ Density of water vapour $\left(\mathrm{kg} / \mathrm{m}^{3}\right)$

$\sigma \quad$ Stefan-Boltzmann constant $\left(5.6710^{-8} \mathrm{~W} /\left(\mathrm{m}^{2} \mathrm{~K}^{4}\right)\right)$

a Absorptivity of the target surface (-)

$A_{p} \quad$ Product area available for sublimation $\left(\mathrm{m}^{2}\right)$

$A_{R_{p}} \quad$ Constant describing $R_{p}$ in function of $l(1 / \mathrm{s})$

$A_{\text {rad }} \quad$ Area of the emitting surface $\left(\mathrm{m}^{2}\right)$

$B_{R_{p}} \quad$ Constant describing $R_{p}$ in function of $l(1 / \mathrm{m})$

$c \quad$ Speed of sound for an ideal gas $(\mathrm{m} / \mathrm{s})$

$F \quad$ View factor (-)

$g \quad$ Gravitational acceleration $\left(9.81 \mathrm{~m} / \mathrm{s}^{2}\right)$

$h \quad$ Height of the spin frozen product $(\mathrm{m})$

$l \quad$ Thickness of dried product layer $(\mathrm{m})$

$L_{t o t} \quad$ Thickness of frozen product layer (m)

$M \quad$ Molecular weight of water $(0.018 \mathrm{~kg} / \mathrm{mol})$

$m \quad$ Mass of sublimed ice (g)

$P_{c} \quad$ Chamber pressure $(\mathrm{Pa})$

$P_{e} \quad$ Electric power $(\mathrm{W})$ 
$P_{\text {rad }}$ Power provided by IR heater to spin frozen vial (W)

$P_{\text {sur }} \quad$ Power provided by surroundings to spin frozen vial (W)

$P_{t o t} \quad$ Total power required for ice sublimation (W)

$P_{w, c} \quad$ Partial water pressure in the drying chamber $(\mathrm{Pa})$

$P_{w, i} \quad$ Vapour pressure at the sublimation interface $(\mathrm{Pa})$

$R \quad$ Gas constant $(8.31 \mathrm{~J} /(\mathrm{K} \mathrm{mol}))$

$R_{p} \quad$ Dried product mass transfer resistance $(\mathrm{m} / \mathrm{s})$

$R_{p, 0} \quad$ Constant describing $R_{p}$ in function of $l(\mathrm{~m} / \mathrm{s})$

$r_{p, i} \quad$ Radius from the centre of the vial to the border of the spin frozen layer $(\mathrm{m})$

$r_{v, i} \quad$ Inner radius of the glass vial (m)

$r_{v, n} \quad$ Radius of the vial neck $(\mathrm{m})$

$T_{c} \quad$ Collapse temperature $(\mathrm{K})$

$T_{e} \quad$ Eutectic temperature $(\mathrm{K})$

$T_{g}^{\prime} \quad$ Glass transition temperature of the maximum freeze-concentrated formulation (K)

$T_{i} \quad$ Product temperature at sublimation front (K)

$T_{r} \quad$ Temperature of the water vapour $(\mathrm{K})$

$T_{v} \quad$ Temperature of the spin frozen vial $(\mathrm{K})$

$T_{i, c r i t}$ Critical product temperature $(\mathrm{K})$

$T_{\text {rad }}$ Temperature of the IR heater $(\mathrm{K})$

$V \quad$ Filling volume $\left(\mathrm{m}^{3}\right)$ 


\section{References}

[1] K. Plumb, Continuous processing in the pharmaceutical industry: Changing the mind set, Chem. Eng. Res. Des. 83 (2005) $730-738$.

[2] C. Vervaet, J. Vercruysse, J. P. Remon, T. De Beer, Continuous Processing of Pharmaceuticals, in: Encyclopedia of Pharmaceutical Science and Technology, Taylor and Francis, New York, 2013.

[3] A. Jungbauer, Continuous downstream processing of biopharmaceuticals, Trends Biotechnol. 31 (2013) 479-492.

[4] T. Jennings, Lyophilization: Introduction and basic principles, Informa healthcare, New York, 2008.

[5] H. R. Constantino, M. J. Pikal, Lyophilization of Biopharmaceuticals, AAPS Press, Arlington (VA), 2004.

[6] A. A. Barresi, R. Pisano, V. Rasetto, D. Fissore, D. L. Marchisio, Model-Based Monitoring and Control of Industrial Freeze-Drying Processes: Effect of Batch Nonuniformity, Drying Technol. 28 (2010) 577-590.

[7] L. De Meyer, P. J. Van Bockstal, J. Corver, C. Vervaet, J. P. Remon, T. De Beer, Evaluation of spin freezing versus conventional freezing as part of a continuous pharmaceutical freeze-drying concept for unit doses, Int. J. Pharm. 496 (2015) 75-85.

[8] P.-J. Van Bockstal, L. De Meyer, J. Corver, C. Vervaet, T. De Beer, Non-contact infrared-mediated heat transfer during continuous freeze-drying of unit doses, J. Pharm. Sci. 106 (2017) 71-82.

[9] J. Corver, Method and system for freeze-drying injectable compositions, in particular pharmaceutical. WO2013036107 (2013).

[10] J. C. Kasper, W. Friess, The freezing step in lyophilization: Physico-chemical fundamentals, freezing methods and consequences on process performance and quality attributes of biopharmaceuticals, Eur. J. Pharm. Biopharm. 78 (2011) $248-263$.

[11] V. R. Koganti, E. Y. Shalaev, M. R. Berry, T. Osterberg, M. Youssef, D. N. Hiebert, F. A. Kanka, M. Nolan, R. Barrett, G. Scalzo, G. Fitzpatrick, N. Fitzgibbon, S. Luthra, L. Zhang, Investigation of design space for freeze-drying: use of modeling for primary drying segment of a freeze-drying cycle., AAPS PharmSciTech 12 (2011) 854-861.

[12] S. L. Nail, J. A. Searles, Elements of Quality by Design in development and scale-up of freeze-dried parenterals, BioPharm Int. 21 (2008) 44-52.

[13] R. Bogner, M. J. Pikal, The Incredible Shrinking Design Space: Using Risk Tolerance to Define Design Space for Primary Drying, Notes of Freeze Drying of Pharmaceuticals and Biologicals Conference, Garmisch-Partenkirchen (2010) $184-198$.

[14] A. Giordano, A. A. Barresi, D. Fissore, On the use of mathematical models to build the design space for the primary drying phase of a pharmaceutical lyophilization process., J. Pharm. Sci. 100 (2011) 311-324.

[15] L. N. Mockus, T. W. Paul, N. A. Pease, N. J. Harper, P. K. Basu, E. A. Oslos, G. A. Sacha, W. Y. Kuu, L. M. Hardwick, J. J. Karty, M. J. Pikal, E. Hee, M. A. Khan, S. L. Nail, Quality by design in formulation and process development for a freeze-dried, small molecule parenteral product: a case study., Pharm. Dev. Technol. 16 (2011) 549-576.

[16] D. Fissore, R. Pisano, A. A. Barresi, Advanced approach to build the design space for the primary drying of a pharmaceutical freeze-drying process, J. Pharm. Sci. 100 (2011) 4922-4933.

[17] R. Pisano, D. Fissore, A. Barresi, P. Brayard, P. Chouvenc, B. Woinet, Quality by design: optimization of a freezedrying cycle via design space in case of heterogeneous drying behavior and influence of the freezing protocol, Pharm. Dev. Technol. 18 (2013) 280-295.

[18] S. Mortier, P. J. Van Bockstal, J. Corver, I. Nopens, K. Gernaey, T. De Beer, Uncertainty analysis as essential step in the 
establishment of the dynamic Design Space of primary drying during freeze-drying, Eur. J. Pharm. Biopharm. 103 (2016) $71-83$.

[19] D. E. Overcashier, T. W. Patapoff, C. C. Hsu, Lyophilization of protein formulations in vials: Investigation of the relationship between resistance to vapor flow during primary drying and small-scale product collapse, J. Pharm. Sci. 88 (1999) 688-695.

[20] S. Rambhatla, R. Ramot, C. Bhugra, M. J. Pikal, Heat and mass transfer scale-up issues during freeze drying: II. Control and characterization of the degree of supercooling., AAPS PharmSciTech 5 (2004) 54-62.

[21] W. Y. Kuu, L. M. Hardwick, M. J. Akers, Rapid determination of dry layer mass transfer resistance for various pharmaceutical formulations during primary drying using product temperature profiles, Int. J. Pharm. 313 (2006) 99-113.

[22] S. M. Patel, S. Chaudhuri, M. J. Pikal, Choked flow and importance of Mach I in freeze-drying process design, Chem. Eng. Sci. 65 (2010) 5716-5727.

[23] R. Bird, W. Stewart, E. Lightfoot, Transport phenoma, John Wiley \& Sons, New York, 2006.

[24] G. F. Nellis, S. A. Klein, Radiation, in: Heat Transfer, Cambridge University Press, Cambridge, 2009.

[25] J. Brenner, Design specifications for wet-bulb aspirator apparatus, Ph.D. thesis, University of Wisconsin-Madison (2010).

[26] J. Vercruysse, M. Toiviainen, M. Fonteyne, N. Helkimo, J. Ketolainen, M. Juuti, U. Delaet, I. Van Assche, J. P. Remon, C. Vervaet, T. De Beer, Visualization and understanding of the granulation liquid mixing and distribution during continuous twin screw granulation using NIR chemical imaging., Eur. J. Pharm. Biopharm.

[27] L. Eriksson, E. Johansson, N. Kettaneh-Wold, J. Trygg, C. Wikström, S. Wold, Multi- and megavariate data analysis part 1: Basic principles and applications, Umetrics, Umeå, 2006.

[28] S. Pieters, Y. Vander Heyden, J. M. Roger, M. D’Hondt, L. Hansen, B. Palagos, B. De Spiegeleer, J. P. Remon, C. Vervaet, T. De Beer, Raman spectroscopy and multivariate analysis for the rapid discrimination between native-like and non-native states in freeze-dried protein formulations, Eur. J. Pharm. Biopharm. 85 (2013) 263-271.

[29] L. Hansen, T. D. Beer, S. Pieters, Y. V. Heyden, C. Vervaet, J. P. Remon, J. P. Montenez, R. Daoussi, Near-infrared spectroscopic evaluation of lyophilized viral vaccine formulations, Biotechnol. Progr. 29 (6) (2013) $1573-1586$.

[30] J. Workman, L. Weyer, Practical Guide to Interpretive Near-Infrared Spectroscopy, CRC Press, Boca Raton (FL), 2007.

[31] M. J. Pikal, Design of freeze-drying processes for pharmaceuticals: practical advice, Pharm. Res. 21 (2) (2004) 191-200. 
Table 1: Nominal values of input parameters for view factor $F$ computation, calculation power contribution by surroundings and calculation dynamic temperature profile

\begin{tabular}{|c|c|}
\hline Parameter & Numerical value \\
\hline \multicolumn{2}{|l|}{ Input parameters view factor computation: } \\
\hline Length radiator & $0.045 \mathrm{~m}$ \\
\hline Width radiator & $0.045 \mathrm{~m}$ \\
\hline Width chamber door & $0.478 \mathrm{~m}$ \\
\hline Height chamber door & $0.523 \mathrm{~m}$ \\
\hline Length drying chamber & $0.557 \mathrm{~m}$ \\
\hline Width drying chamber & $0.478 \mathrm{~m}$ \\
\hline Height drying chamber & $0.523 \mathrm{~m}$ \\
\hline Length shelf & $0.453 \mathrm{~m}$ \\
\hline Width shelf & $0.300 \mathrm{~m}$ \\
\hline Outer radius vial $r_{v, o}$ & $0.0119 \mathrm{~m}$ \\
\hline Vial height $h$ & $0.040 \mathrm{~m}$ \\
\hline Distance between centre cylinder and IR heater & $0.040 \mathrm{~m}$ \\
\hline Distance between centre cylinder and chamber door & $0.150 \mathrm{~m}$ \\
\hline Distance between centre cylinder and left chamber wall & $0.270 \mathrm{~m}$ \\
\hline Distance between centre cylinder and top shelf & $0.220 \mathrm{~m}$ \\
\hline Distance between centre cylinder and bottom shelf & $0.070 \mathrm{~m}$ \\
\hline Number of simulated rays (Monte Carlo simulation) & $10^{6}$ \\
\hline \multicolumn{2}{|l|}{ Input parameters power contribution surroundings: } \\
\hline Emissivity quartz glass $(\mathrm{IR}$ heater $) \epsilon$ & 0.93 \\
\hline Emissivity stainless stee $\epsilon$ & 0.83 \\
\hline Emissivity acrylic glass $\epsilon$ & 0.86 \\
\hline Temperature chamber door & $288.75 \mathrm{~K}$ \\
\hline Temperature chamber wall (left) & $288.95 \mathrm{~K}$ \\
\hline Temperature chamber wall (right) & $289.15 \mathrm{~K}$ \\
\hline Temperature chamber wall (back) & $287.45 \mathrm{~K}$ \\
\hline Temperature shelves & $263.15 \mathrm{~K}$ \\
\hline \multicolumn{2}{|c|}{ Specific input parameters for calculation dynamic temperature profile: } \\
\hline Inner radius vial $r_{v, i}$ & $0.0109 \mathrm{~m}$ \\
\hline Inner radius vial neck & $0.0063 \mathrm{~m}$ \\
\hline Product resistance parameter $R_{p, 0}$ & $1.8510^{4} \mathrm{~m} / \mathrm{s}[21]$ \\
\hline Product resistance parameter $A_{R_{p}}$ & $1.2010^{7} 1 / \mathrm{s}[21$ \\
\hline Product resistance parameter $B_{R_{p}}$ & $-6.76101 / \mathrm{m}[21$ \\
\hline Filling volume $V$ & $3.90 \mathrm{~mL}$ \\
\hline Volume fraction ice $\phi$ & 1 \\
\hline View factor $F$ & 0.1484 \\
\hline Critical product temperature $T_{i, c r i t}$ & $240.15 \mathrm{~K}$ \\
\hline
\end{tabular}


Table 2: Overview of the comparison between the computed and experimental primary drying time; Computed primary drying time: 50 minutes

\begin{tabular}{ccc} 
Experiment & $\begin{array}{c}\text { Experimental primary } \\
\text { drying time (mm:ss) }\end{array}$ & $\begin{array}{c}\text { Ratio experimental to computed } \\
\text { primary drying time }(-)\end{array}$ \\
\hline 1 & $45: 00$ & 0.90 \\
2 & $47: 19$ & 0.95 \\
3 & $46: 00$ & 0.92 \\
4 & $46: 50$ & 0.94 \\
5 & $43: 32$ & 0.87 \\
6 & $44: 09$ & 0.88 \\
\hline
\end{tabular}




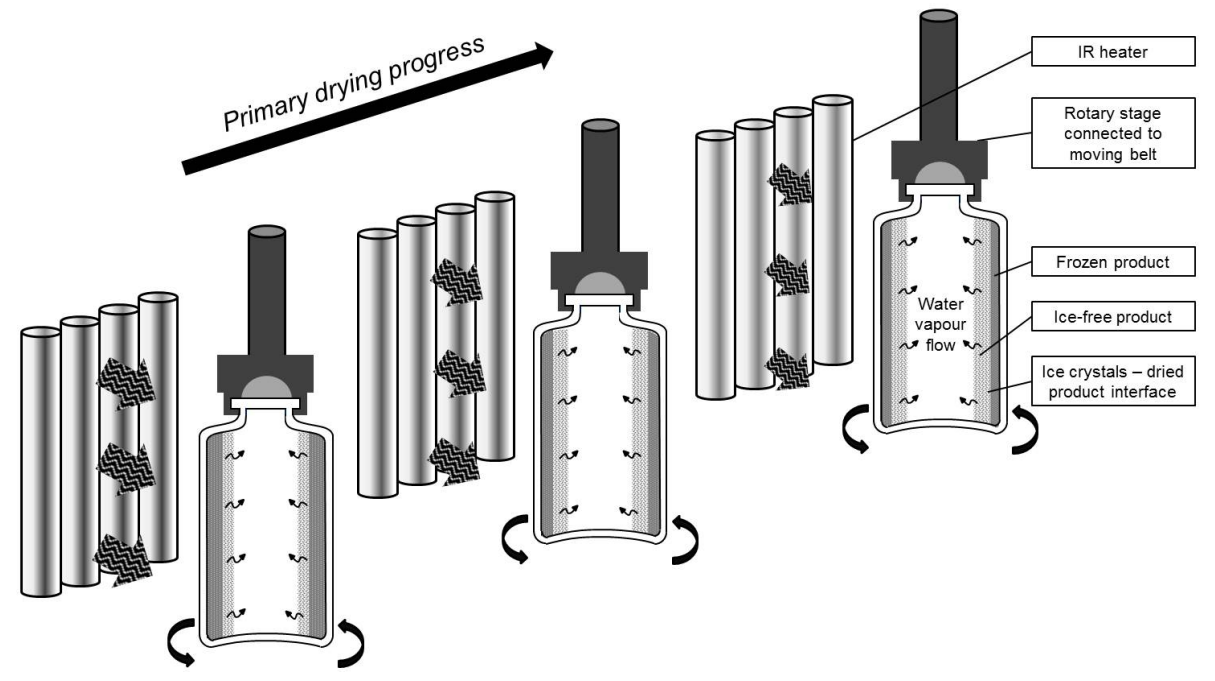

Figure 1: Illustration of the IR assisted continuous primary drying of spin frozen vials, rotating along their longitudinal axis in front of individual IR heaters 


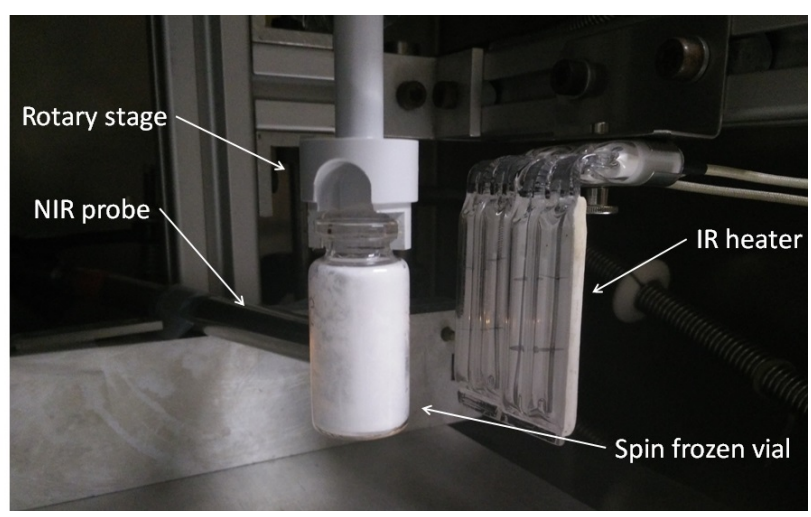

Figure 2: Experimental drying set-up with in-line NIR monitoring 


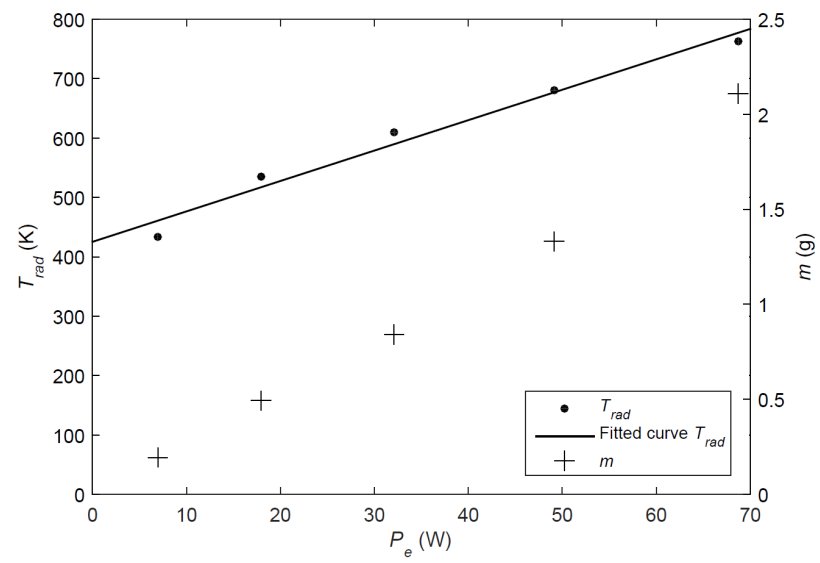

Figure 3: Mass of sublimed ice $m$ and temperature of $\mathrm{IR}$ heater $T_{\text {rad }}$ in function of electric power $P_{e}$ 


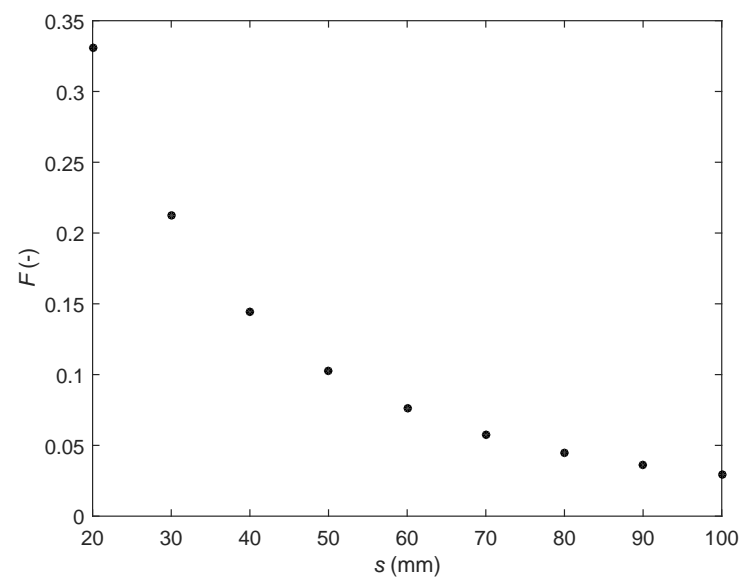

Figure 4: View factor $F$ in function of the distance between the $\mathrm{IR}$ heater and the centre of the spin frozen vial $s$ 


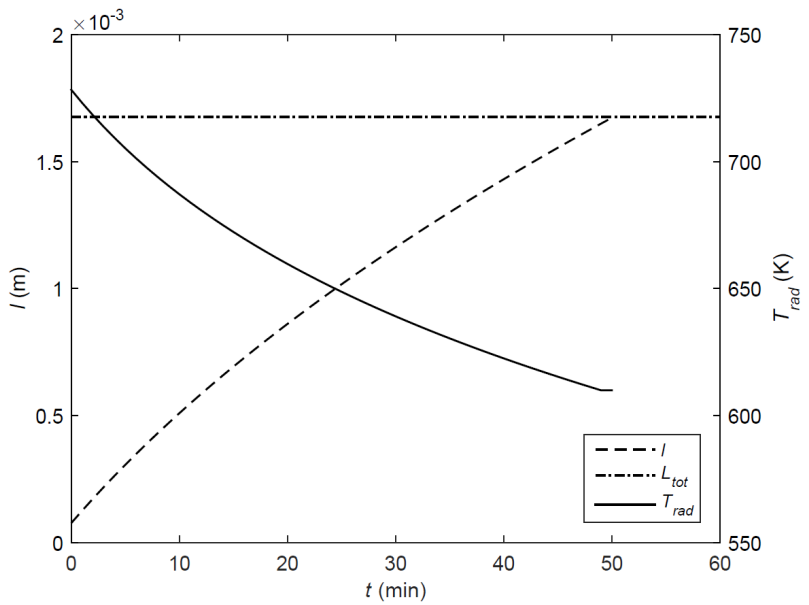

Figure 5: IR heater temperature $T_{\text {rad }}$ and dried layer thickness $l$ in function of primary drying time $t$; the black dash-dotted line represents the total layer thickness of the spin frozen cake $L_{t o t}$ 


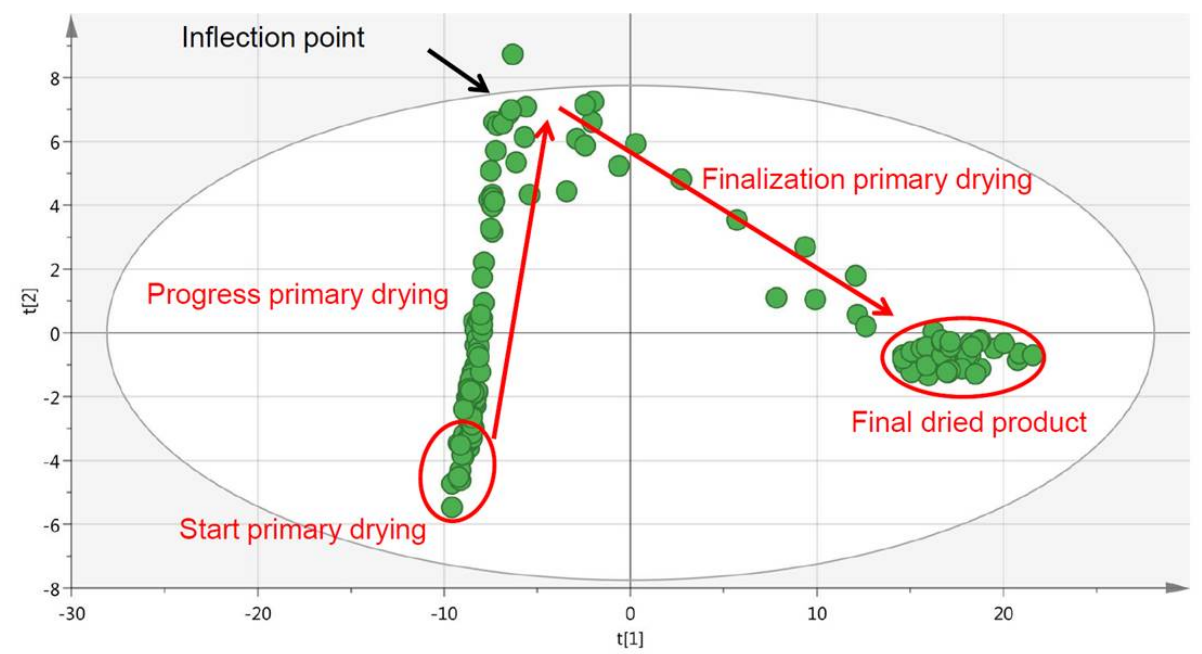

Figure 6: Scores scatter plot including the explanation of the chronological trends; each point represents 1 NIR spectrum 


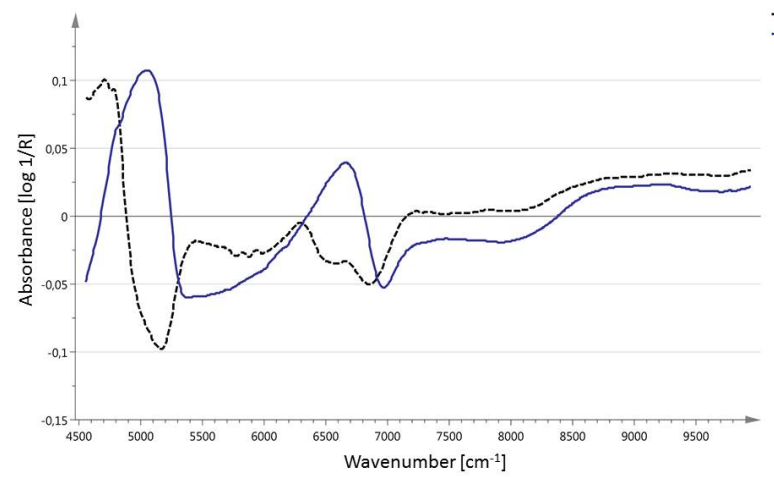

Figure 7: Loading line plot of $\mathrm{PCl}$ (black dotted) and $\mathrm{PC}$ (blue solid) 

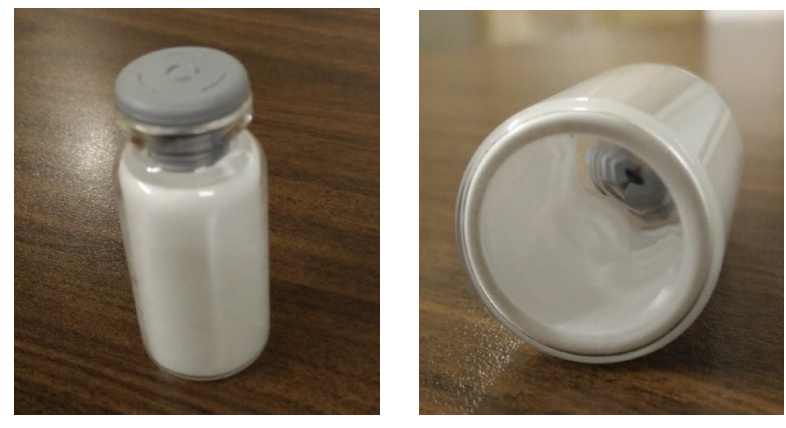

Figure 8: Product appearance of final dried product yielded after the validation runs 